\title{
Source Localization accuracy in an animal model
}

\author{
L. Flemming *, J. Haueisen *, U. Tenner*, F. Gießler*, M. Eiselt**
}

Biomagnetic Center, Department of Neurology, Jena University, Jena, Germany *

Department of Pathophysiology, Jena University, Jena, Germany **

\section{Introduction}

Source localization in the human brain based on EEG and MEG data becomes more and more important in the field of clinical research and routine. Thus, information about source localization accuracy is indispensable. Source localization accuracy has been tested with various artificial source and volume conductor models. However, there are a number of limitations inherent to these artificial models. Thus, we address source localization accuracy with an animal model. Furthermore, most investigations have been performed with artificial and physiological sources of a tangential orientation. However, 20 percent of the sources of the human brain have a radial orientation. Hence, an animal model with a lissencephalic cerebrum (flat cortical surface) with radial oriented physiological sources would take this into account.

\section{Methods}

The investigations were performed with a physiological and an artificial source model. Rabbits (White New Zealand) were used for this investigation because of their lissencephalic brain.

Two different nerves with well defined cortical representations of about 1 to $1.5 \mathrm{~mm}^{2}$ were stimulated for the physiological source model. The spatial distance between the cortical areas is about 2 to $3 \mathrm{~mm}$ [1].

The dipole for the artificial source model had to have a length equal to the extension of the activated pyramidal cells within the somatosensory cortex of the rabbit. The length varied between 1 to $1.5 \mathrm{~mm}$ [1]. The diameter had to be as small as possible. We used a dipole with a length of 1.2 and a diameter of $0.3 \mathrm{~mm}$.

New Zealand white rabbits with an age of approximately 6 month and a weight of approximately $5 \mathrm{~kg}$ were anaesthetized with a combination of Ketamin and Xylazine. Initially, the anaesthetic agents were injected in the muscle of the left upper hindpaw and later in the left femoral vein. The cannulation of the femoral artery on the same side was used for monitoring of physiological parameters e.g. blood pressure and blood withdrawal for blood gas analysis. Xylocain as local anesthetic agent was used for every surgical approach. Animals were kept normothermic and were allowed to breath spontaneously. Small silver stimulation electrodes were implanted and placed directly on the right median and right tibial nerve.
The position of the electrode was defined by anatomical structures. A current pulse was used for the stimulation and varied between 0.2 to $0.5 \mathrm{~mA}$. The current was increased in strength until a muscle contraction was visible.

The nerve stimulation modalities were varied in interstimulus interval $(100 \mathrm{~ms}, 500$ and $1000 \mathrm{~ms})$ and trials averaged $(512,1024,2048)$.

The artificial dipole was placed within the same region of the brain where the physiological dipole was found.

A Current of $10 \mu \mathrm{A}$ and a frequency of $5 \mathrm{~Hz}$ were used.

The scalp and skull were removed in a rectangle area of $7 \times 9 \mathrm{~mm}$ to place the ECoG electrode on the surface of the cortex.

After all surgical preparations have been finished the rabbit was placed in a stereotactic frame inside a magnetically shielded room. Anatomical markers of the skull, the position of the ECoG electrode, and the SQIUD-MEG system were digitized.

A 16 channel micro SQUID-MEG system was used for recording the magnetic field, which is described more in detail by Nowak et al [2]. The ECoG was recorded by a grid of $4 \times 4$ electrodes simultaneously over the contralateral hemisphere of the stimulated nerves. The diameter of each single electrode was $0.25 \mathrm{~mm}$ and spatial distance between adjacent electrodes was $1.25 \mathrm{~mm}$.

Data were recorded with a sampling rate of $2 \mathrm{kHz}$, a high pass filter of $0.3 \mathrm{~Hz}$ and a low pass filter of $300 \mathrm{~Hz}$.

A BEM model and a single dipole was used for the source localization.

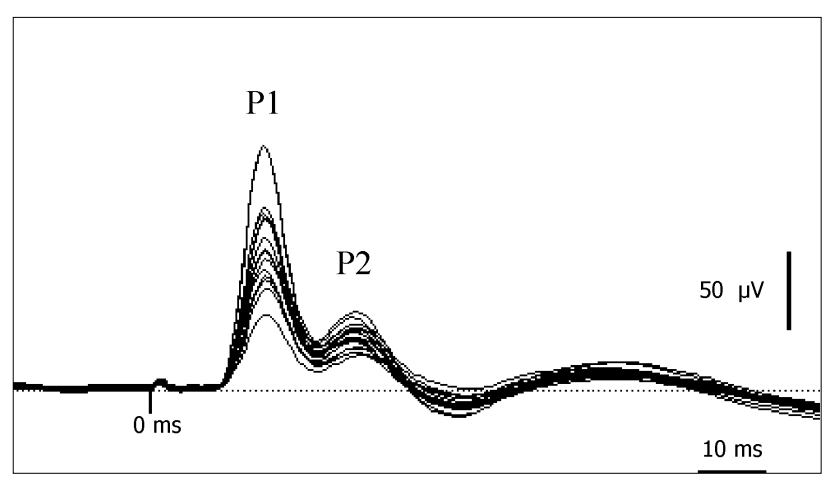

Fig.1a Electric signal of median nerve with main peaks named as P1 and P2, time range : -20 to $100 \mathrm{~ms}$, 2048 averages 


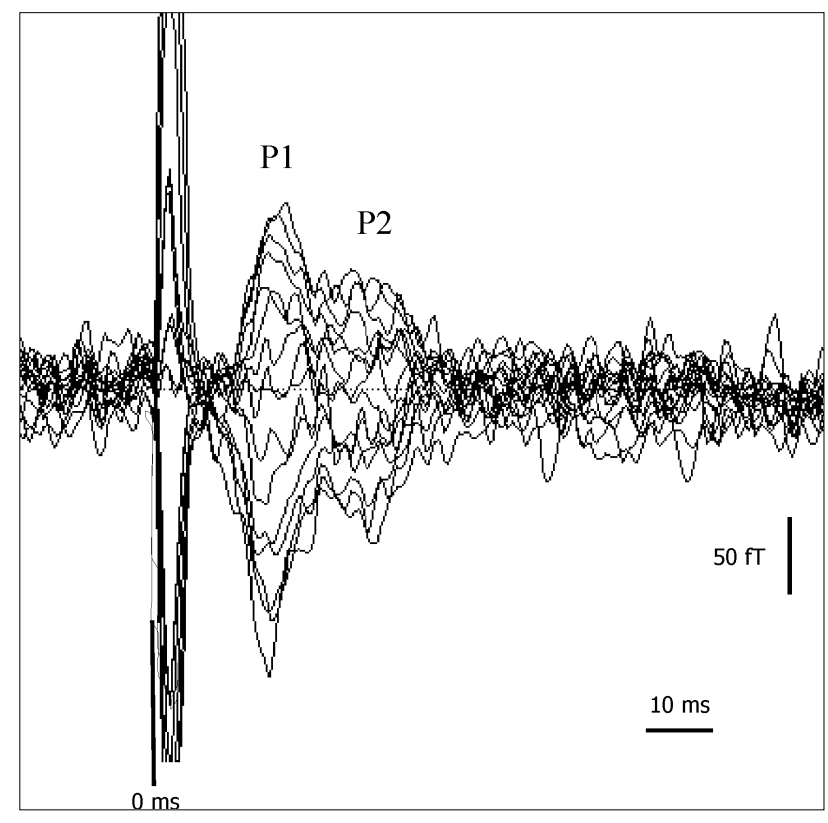

Fig. 1b Magnetic signal of median nerve with main peaks named as $\mathrm{P} 1$ and $\mathrm{P} 2$, time range : -20 to $100 \mathrm{~ms}$, 2048 averages, stimulation artifact ( 0 to $8 \mathrm{~ms}$ )

A T1 weighted MR data set was the basis for the BEM model. It had a field of view of $80 \times 80 \mathrm{~mm}$, a slice thickness of $0.5 \mathrm{~mm}$ and a pixel size of $0.31 \times 0.31 \mathrm{~mm}$. A Siemens Magnetom Vision 2000 was used for the measurement.

The BEM model consisted of 4673 points with a distance of $0.45 \mathrm{~mm}$ between adjacent points in the crucial region of the brain.
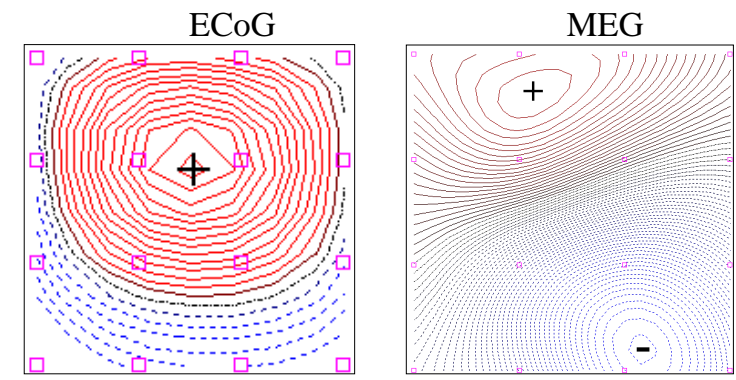

a
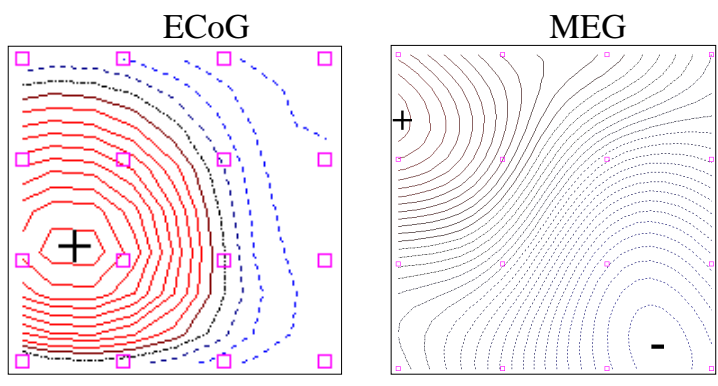

b

Fig. 2 Electric field pattern and magnetic field pattern of median nerve (a) and tibial nerve (b), Increment $=5 \mu \mathrm{V} / 5 \mathrm{fT}$

Distance between ECoG electrodes $1.25 \mathrm{~mm}$ and MEG pick-up coils $8.4 \mathrm{~mm}$ (rectangles in field pattern)

\section{Results}

The electric and magnetic signal of the physiological stimulation of the median and tibial nerve yielded two main peaks named as P1 and P2 (Fig. 1). The artifact in the magnetic signal was caused by the stimulation electrodes.

We have focused our investigations to $\mathrm{P} 1$ which is known as the first answer in the somatosensory cortex S1 and which is not affected by any cortical activities.

There was a difference of about $4 \mathrm{~ms}$ in the latency of both nerves. The averaged latency of the median nerve was $17 \mathrm{~ms}$ and of the tibial nerve $21 \mathrm{~ms}$. The inter- and intraindividual variation was in a range of $5 \mathrm{~ms}$.

The physiological stimulation yielded a clear dipolar field pattern for the magnetic and a monopolar field pattern for the electric signal (Fig. 2). The differences in the localization of both nerves are obvious (Fig. 2). Data processing of the raw signal were performed for the source localization. It consisted of a baseline correction, a Wiener filter and an artifact suppression. Both signals were used for the source localization.

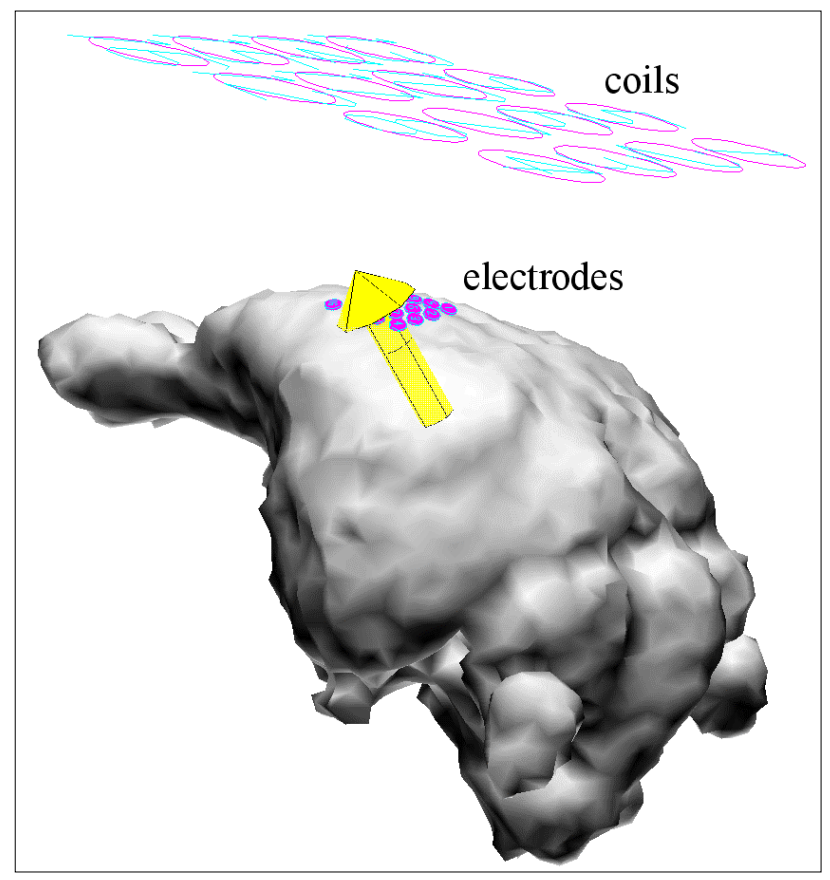

Fig. 3 Result of source localization of the physiological stimulation of the median nerve

The result of the source localization is a dipole with a radial orientation within in the expected cortical area (Fig. 3) for the median and tibial nerve. The localized sources were within an accuracy of $1 \mathrm{~mm}$ in the expected cortical areas. The spatial distance between both nerves was in a range of $2.5+/-0.5 \mathrm{~mm}$ comparable with the anatomical difference.

Both the artificial dipole in a sphere (Fig. 4a) and in the animal model (Fig. 4b) yielded a clear dipolar field pattern. 


\section{Discussion}

We found the rabbit model suitable for the investigations of source localization accuracy, especially for the investigations of radially oriented sources. The advantage compared with the work by Melcher and Cohen [3] is that we used a physiological source. Hence, we simulated a more realistic situation because we did not destroy any tissue in the brain. The tissue damage would effect e.g. conductivity parameters which are important for the source localization.

It was possible to achieve an accuracy of $1 \mathrm{~mm}$ by the use of the electric and the magnetic signals simultaneously. However, the source localization was mainly influenced by the ECoG due to the higher SNR. The accuracy obtained by the use of either the electric or the magnetic signals remained to be investigated in a further study.

The calculation of source localization accuracy was based on the anatomical maps by Gould [1]. An uncertainty in this case is the alteration in the size of receptive fields in the somatosensory cortex between the anesthetized and unanesthetized state which was reported by McKenna et al. [4]. They observed a larger field in the unanesthetized state. However, since we use a single dipole approach only in homogeneous changes in the size of rezeptive field will produce dipole shifts. Homogeneous enlargement will produce increased dipole amplitudes.

The combination of Ketamin and Xylazine with minimal influences to the SEF/SEP proved to be a good choice for a high SNR. The only effect which we have observed was a delay in the latency. A higher dose increased the latency notably.

Interstimulus intervals of $100 \mathrm{~ms}$ produced a lower signal amplitude and different structure of the signal as compared to interstimulus intervals of 500 and $1000 \mathrm{~ms}$. The difference in the signal amplitude between 500 and $1000 \mathrm{~ms}$ was not significant and the signal structure was similar.
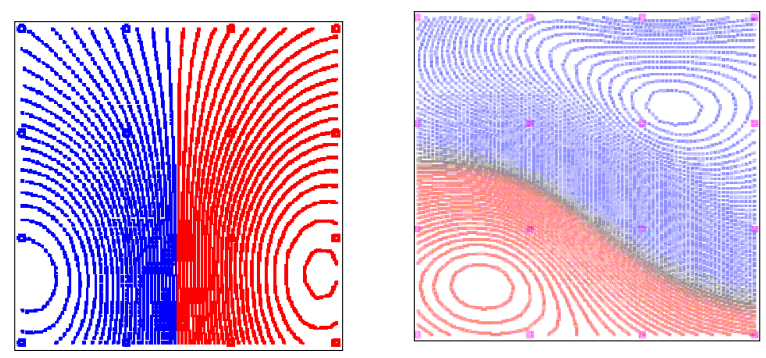

Fig. 4 Magnetic field pattern of the physical dipole of tangential orientation in a sphere (left) and in a rabbit (right)

\section{References}

[1] Gould, H.J., III, Body Surface Maps in the Somatosensory Cortex of Rabbit, The Journal of Comparative Neurology, 1986, 243: 207-233.

[2] Nowak, H., Gießler,F., Huonker, R., Haueisen, J., Röther, J., Eiselt, M., A 16-channel SQUID-device for biomagnetic investigations of small objects, Medical Engineering \& Physics, 1999, 21: 563-568.

[3] Melcher, J.R., Cohen, D., Dependence of the MEG an dipole orientation in the rabbit head, Electroencephalography and clinical Neurophysiology, 1988, 70: 460-472.

[4] McKenna, T.M., Whitsel, B.L., Dreyer,D.A., and Metz,C.B., Organization of the cat anterior parietal cortex: relations among cytoarchitecture, single neuron functional properties and interhemispheric connectivity, Journal of Neurophysiology, 1981, 45: 667-697.

\section{Acknowledgements}

This work was supported by the European Community ( project number: IST 1999-10378). 